Check for updates

Cite this: RSC Adv., 2019, 9, 39945

\title{
Core-shell magnetic nano-powders with an excellent decolorization effect on dye wastewater
}

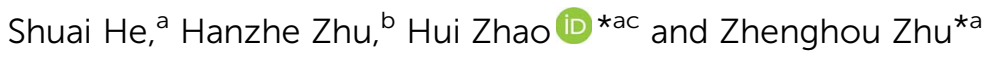

In recent years, zero-valent nano-iron ( $\mathrm{nZVI}$ ) has received extensive attention due to its excellent decolorization effect on dye wastewater. In this paper, zero-valent nano-iron-nickel (nZVIN) powders were prepared by a simple, efficient and non-polluting method. The powder has a unique core-shell structure and excellent oxidation resistance. Hence the problem that nZVI powders are easily oxidized and difficult to store is solved. Due to the addition of $\mathrm{Ni}$, the magnetic properties of the nZVIN powders are enhanced, which facilitates the recycling of the powders using a magnetic field after sewage treatment. In the decolorization treatment of dye wastewater simulated with Congo red (CR) dye, nZVIN powders can maintain a removal rate of more than $90 \%$ for $\mathrm{CR}$ solutions with different $\mathrm{pH}$ values (7.011.5) and an initial dye concentration $\left(50-200 \mathrm{mg} \mathrm{L}^{-1}\right.$ ). The research results show that nZVIN powders have broad application prospects in the treatment of azo dye wastewater.
\end{abstract}

Received 30th September 2019 Accepted 19th November 2019

DOI: 10.1039/c9ra07937h

rsc.li/rsc-advances free radicals to remove dyes during adsorption. ${ }^{6}$ However, the adsorption effect of this method is single, and it is effective only for a specific one or several dyes, and it is difficult to cope with complicated and diverse polluted wastewater, meanwhile the adsorbent treatment after adsorption is also a problem. ${ }^{7}$ The second method is the membrane filtration, but the membrane as a filter is easily contaminated, and requires frequent replacement, and its cost is expensive to use. The third one is the biodegradation method. Bioremediation, as an ecologically friendly method, has attracted extensive attention because of its advantages of degrading dye wastewater and improving soil. ${ }^{8}$ In practical applications, the degradation effect on dye wastewater is relatively common, and it is not suitable for treating dye wastewater with complex composition because microorganisms are vulnerable to environmental influences and instability.

Zero-valent iron (nZVI) in dye wastewater treatment is increasingly valued because of its chemical nature, low price, and strong degradation and reduction ability for dye wastewater. ${ }^{9}$ K. V. G. Ravikumar prepared nZVI under anaerobic conditions using anaerobic sludge granules and obtained a 99\% removal rate for the methyl orange dye by nZVI obtained. ${ }^{\mathbf{1 0}}$ Zhang Lian'an prepared S-ZVI by sulfonation, and improved the removal rate of azo dye. ${ }^{11}$ Akeem Adeyemi Oladipo prepared $\mathrm{CoO}-\mathrm{NiFe}_{2} \mathrm{O}_{4}$ catalyst by coprecipitation method and calcination method, and studied its degradation effect and decolorization mechanism on dye wastewater in detail. ${ }^{12}$ Although the above preparation method solves the problem of nZVI preparation, it is still to be solved that nZVI is easy to oxidize in the process of use and storage, difficult to be stored, and difficult to be recovered when dispersed in the solution.

In this paper, in order to solve the above problem of using nZVI, the nickel element was added to the pure iron powders to 
form $\mathrm{FeNi}_{3}$ alloy phase. Compared with the previous one, the reaction preparation process is simple and environmentally friendly, and its oxidation resistance and magnetic properties have been significantly enhanced to facilitate preservation and recycling. Spherical nano-scale zero-valent iron-nickel (nZVIN) powders were prepared by chemical reduction method, and a series of characterizations were carried out. It was found that the shape of particle size was uniform and controllable. By alloying with the addition of Ni element, oxidation resistance of the powders is enhanced, and magnetic properties are also improved. Meanwhile, the problems of zero-grade iron in storage, transportation and recycling are solved. More importantly, the added nickel element has a catalytic effect on the generation of atomic hydrogen in the solution by zero-valent iron, which can promote the reduction and decolorization of the dye by zero-valent iron. ${ }^{13}$ In the decolorization treatment of dye wastewater simulated by Congo red (molecular formula $\mathrm{C}_{32} \mathrm{H}_{22} \mathrm{~N}_{6} \mathrm{Na}_{2} \mathrm{O}_{6} \mathrm{~S}_{2}$, structural formula shown in Fig. 1), nZVIN powders preserving for one year can maintain a removal rate of more than $90 \%$ for CR solutions in a wide $\mathrm{pH}$ value (7.0-12.0). And the nZVIN powders in the solution after treatment can be removed by simple magnetic separation.

\section{Experiment}

\subsection{Drugs and characterization}

All the solutions in this paper were prepared with deionized water. All chemical materials were ones of analytical grade, such as, ferrous sulfate heptahydrate $\left(\mathrm{FeSO}_{4} \cdot 7 \mathrm{H}_{2} \mathrm{O}\right)$, nickel sulfate hexahydrate $\left(\mathrm{NiSO}_{4} \cdot 6 \mathrm{H}_{2} \mathrm{O}\right)$, hydrazine hydrate $\left(\mathrm{N}_{2} \mathrm{H}_{4} \cdot \mathrm{H}_{2} \mathrm{O}\right)$, sodium hydroxide $(\mathrm{NaOH})$, hydrochloric acid $(\mathrm{HCl})$, Congo red (CR) and alcohol.

The phase identification of nZVIN powders was performed by X-ray diffraction (XRD) on a Bruker-axe D8 ADVANCE X-ray diffract meter with $\mathrm{CuK} \alpha$ radiation. The test conditions were that tube voltage was $40 \mathrm{kV}$, the current was $40 \mathrm{~mA}$, and the step was $0.02^{\circ}$. Particle microstructures and morphologies were observed by employing a field emission scanning electron microscopy (JSM-6701F(JEOL)) and transmission electron microscope (Hitachi 7650B). The hysteresis loop was tested by PPMS-9 physical property test system (American quantum design company), in which the sweep speed was 100 Oe $\mathrm{s}^{-1}$ and the test temperature was $300 \mathrm{~K}$.

The absorbance of the CR dye was measured by an ultraviolet spectrophotometer with a measurement step of $2 \mathrm{~nm}$ and a scanning range of 400-600 $\mathrm{nm}$. The infrared spectra of samples before and after treatment were measured by Nicolet

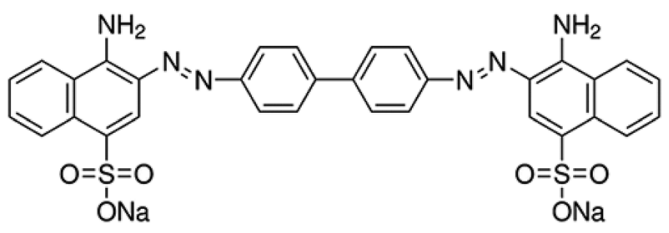

Fig. 1 Congo red molecular formula.

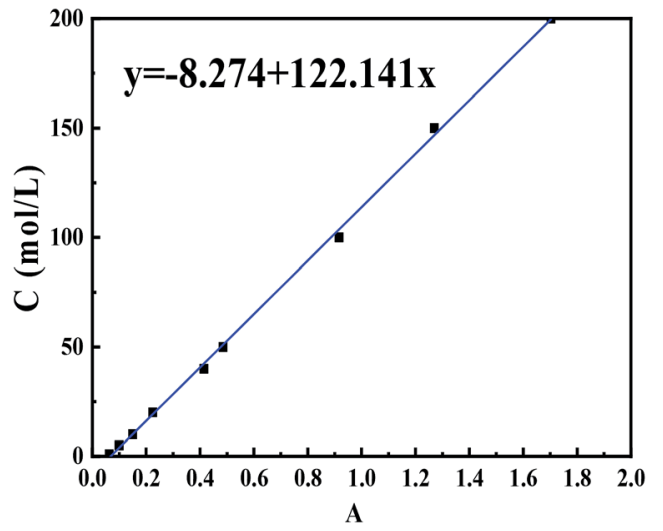

Fig. 2 Concentration-absorbance curve of CR solution.

380 Fourier transform spectrometer (resolution $2 \mathrm{~cm}^{-1}$ ) by $\mathrm{KBr}$ compression method.

\subsection{Preparation of $\mathrm{nZVIN}$ powders}

$\mathrm{FeSO}_{4} \cdot 7 \mathrm{H}_{2} \mathrm{O}$ of $10.5 \mathrm{~g}$ and $\mathrm{NiSO}_{4} \cdot 6 \mathrm{H}_{2} \mathrm{O}$ of $10 \mathrm{~g}$ were dissolved in water solution of $200 \mathrm{~mL}$, and the solution was heated in a water bath, and keep the temperature at $85{ }^{\circ} \mathrm{C}$. Then $\mathrm{NaOH}$ of $6 \mathrm{~g}$ was added into the mixture, and stirred at a high-speed of $1500 \mathrm{rpm}$. When gray-green precipitates appeared in the mixture, $\mathrm{N}_{2} \mathrm{H}_{4} \cdot \mathrm{H}_{2} \mathrm{O}$ of $11.25 \mathrm{~g}$ was added, and the solution reacted vigorously with stirring for about $30 \mathrm{~min}$, and the reaction process was associated with colorless and irritating gas volatile out, at last black powders were generated. After completion of the reaction, the resultant was washed three times with deionized water and absolute ethanol to remove residual starting materials and reagents. Finally, the nZVIN powders were obtained by magnetic separation and drying.

\subsection{Decolorization experiment}

The azo dye wastewater was simulated using CR dye. CR of $1 \mathrm{~g}$ $\mathrm{L}^{-1}$ was prepared firstly as a stock solution with deionized water, and then configured to different concentrations of CR standard solution. The absorbance of different concentrations

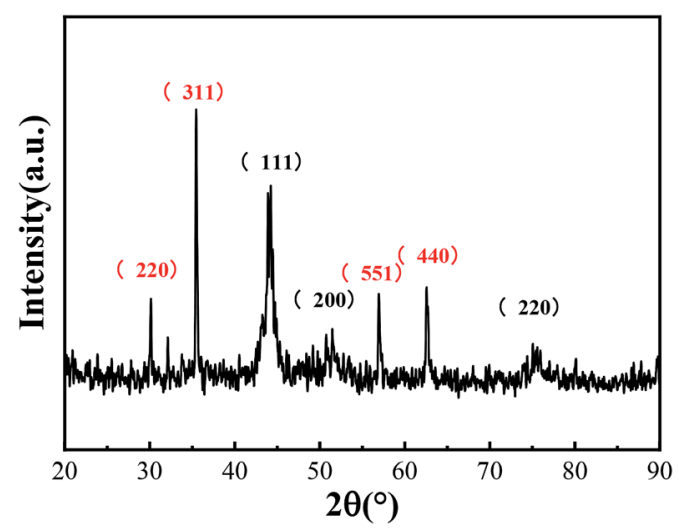

Fig. 3 XRD spectrum of nZVIN powders. 

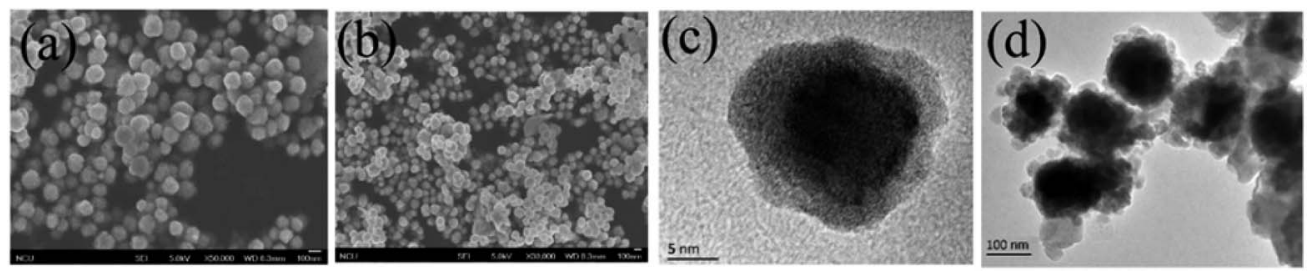

Fig. 4 Micrographs of nZVIN powders of nZVIN powders.

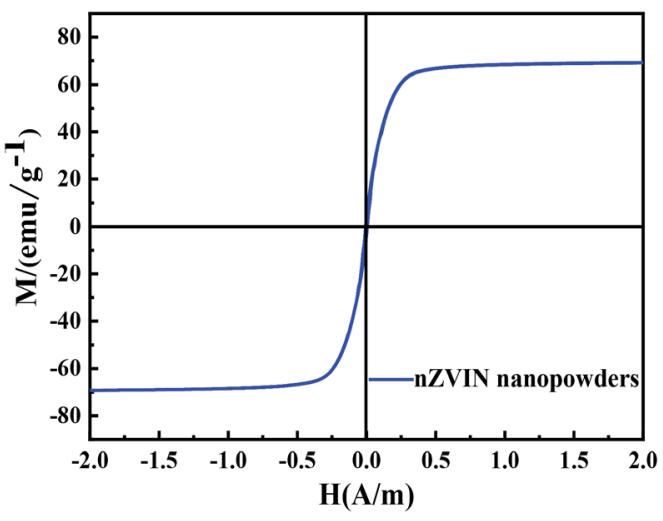

Fig. 5 Hysteresis loop of nZVIN powders.
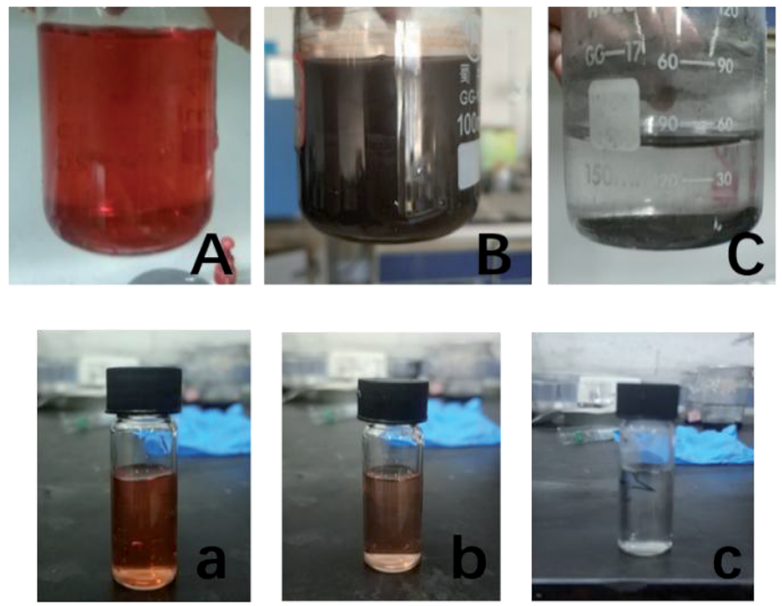

Fig. 6 CR solution color change process after nZVIN powders being added $((A-C)$ pre and post reaction solution. $(a-c)$ Test sample before and after reaction).

of Congo red solution was measured by a UV spectrophotometer at the characteristic absorption peak of $\mathrm{CR}(\lambda=497 \mathrm{~nm})$ to obtain a CR solution concentration-absorbance curve (Fig. 2). The nZVIN powders of $0.1 \mathrm{~g}$ were added into $100 \mathrm{~mL} \mathrm{CR}$ solution of different concentration or different initial $\mathrm{pH}$ value, and the mixture was sonicated in ultrasound. Suspension of $3 \mathrm{~mL}$ was taken at regular intervals, and the supernatant was taken after centrifugation, and the absorbance was measured by an ultraviolet spectrophotometer, and then the CR concentration was estimated from the curve of Fig. 2.
At time $t(\mathrm{~min})$, the removal amount $Q_{t}$ of CR can be calculated by the eqn (1), and the removal rate $R_{t}$ of CR is calculated by the eqn (2).

$$
\begin{gathered}
Q_{t}=\left(C_{0}-C_{t}\right) / m V \\
R_{t}=\left(C_{0}-C_{t}\right) / C_{0} \times 100 \%
\end{gathered}
$$

where, $Q_{t}$ is the amount of $C_{\mathrm{R}}$ removed of $1 \mathrm{~g}$ nZVIN powders, $R_{t}$ is the removal rate of $C_{\mathrm{R}}$ of $1 \mathrm{~g}$ nZVIN powders, $C_{0}$ and $C_{t}$ are the initial and the time of $t$ concentration of $C_{\mathrm{R}}, m$ is the mass of nZVIN powders, and $V$ is the volume of solution.

\section{Results and discussion}

Hydrazine hydrate has both oxidizability and reducibility. The oxidation potential of $\mathrm{Fe}^{2+}$ to $\mathrm{Fe}^{3+}$ is $0.66 \mathrm{~V}$, and the reduction potential of reduction to zero valence iron is $0.283 \mathrm{~V}$. Therefore, $\mathrm{Fe}^{2+}$ is difficult to reduce to obtain zero-valent iron generally. But, when $\mathrm{Ni}^{2+}$ and $\mathrm{Fe}^{2+}$ coexist, part of $\mathrm{Fe}^{2+}$ and $\mathrm{Ni}^{2+}$ can be reduced in a ratio of $1: 3$. Hence, nZVIN powders were prepared by adding Ni element in this experiment. ${ }^{14}$ At the beginning of the reaction, the molar ratio of $[\mathrm{Ni}]$ to $[\mathrm{Fe}]$ is $1 / 1$, and $\mathrm{FeNi}_{3}$ nuclei were first formed, and gradually grew until Ni element consumed, and a part of the remaining $\mathrm{Fe}^{2+}$ were oxidized to $\mathrm{Fe}^{3+}$, which combined with [O] to form $\mathrm{Fe}_{3} \mathrm{O}_{4}$. As can be seen from the XRD pattern (Fig. 3), the phase composition of the nZVIN powder phase is $\mathrm{Fe}_{3} \mathrm{O}_{4}$ (represented by the red crystal face index) and $\mathrm{FeNi}_{3}$ (represented by the black crystal face index).

The unique size and morphology of nZVIN powders can be observed from Fig. 4. The nZVIN powder has a particle size of about $60 \mathrm{~nm}$ and is spherical (Fig. 4(a) and (b)). This special size and spherical shape make the nZVIN powders have a large specific surface area and surface energy, which contributes to enhance its reactivity in solution.

It can be seen from the TEM image (Fig. 4(c) and (d)) that the nZVIN powder consists of two layers of different component, the core layer has a high Ni content and a darker color, the outer layer has a lower Ni content and a lower color, which confirms that nZVIN powders have a core-shell structure. In addition, since the $[\mathrm{Ni}] /[\mathrm{Fe}]$ molar ratio is $1 / 1$ at the reaction, $\mathrm{FeNi}_{3}$ nuclei are formed in the initial stage of the reaction, and as the reaction increases, [Ni] has less residual content than [Fe], the surface of the powders formed at the end of the reaction have a large number of defects and lattice distortion, which is favorable for adsorption and reaction with CR. 

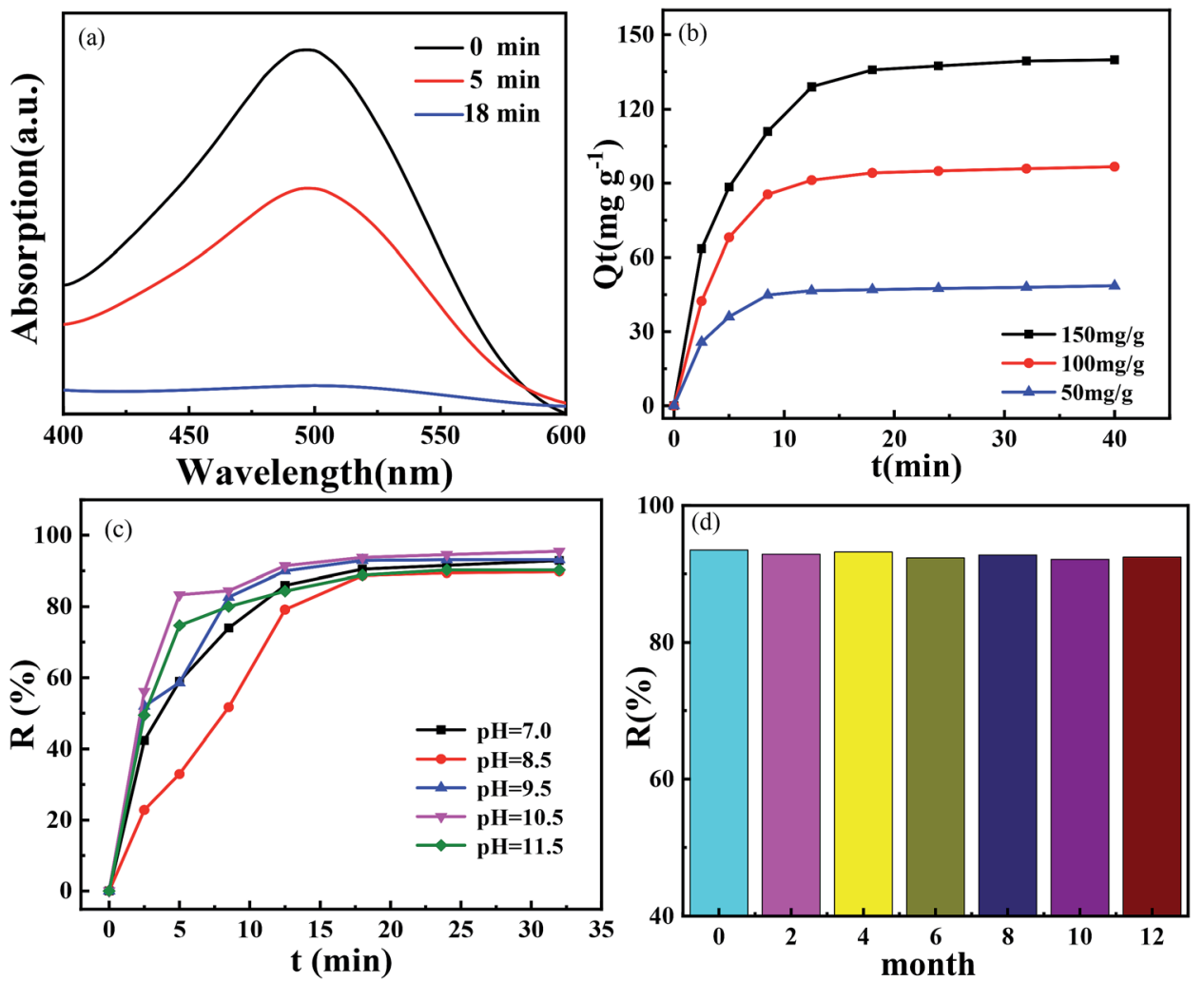

Fig. 7 Removal effect of CR by nZVIN powders under different conditions (a) ultraviolet spectrums of solution in the process of CR removal (b) unit removal of CR under different initial nZVIN concentrations (c) removal rate of CR by nZVIN powders under different $\mathrm{pH}$ conditions (d) removal rate of CR by $\mathrm{nZVIN}$ powders stored in open air for different times.

In addition, magnetic properties are also key factors determining the application of nZVIN powders. The magnetic properties can be significantly enhanced by the addition of Ni. The hysteresis loop of the nZVIN powders measured at room temperature and a kind of typical S-type hysteresis loop (Fig. 5). Hence the nZVIN powder is a typical ferromagnetic material. The nZVIN powder has a high $M_{\mathrm{s}}\left(69.8 \mathrm{emu}^{-1}\right)$, a low $H_{\mathrm{c}}$ $\left(0.0047 \mathrm{~A} \mathrm{~m}^{-1}\right)$ and a $M_{\mathrm{r}}\left(4.79 \mathrm{emu} \mathrm{g}^{-1}\right)$. Due to the good magnetic properties, the nZVIN powders can respond quickly under the action of external magnetic field, which can solve the problem of the recovery of powders after actual sewage treatment, which is favorable for adsorption and reaction with CR.

$150 \mathrm{mg} \mathrm{L}^{-1} \mathrm{CR}$ solution was used to simulate azo dye wastewater with CR as target pollutant. The nZVIN powders of $0.1 \mathrm{~g}$ were added for decolorization experiment. The color of the solution was red at the beginning, then the color turned dark red after nZVIN powders had been put in solution. Finally, the
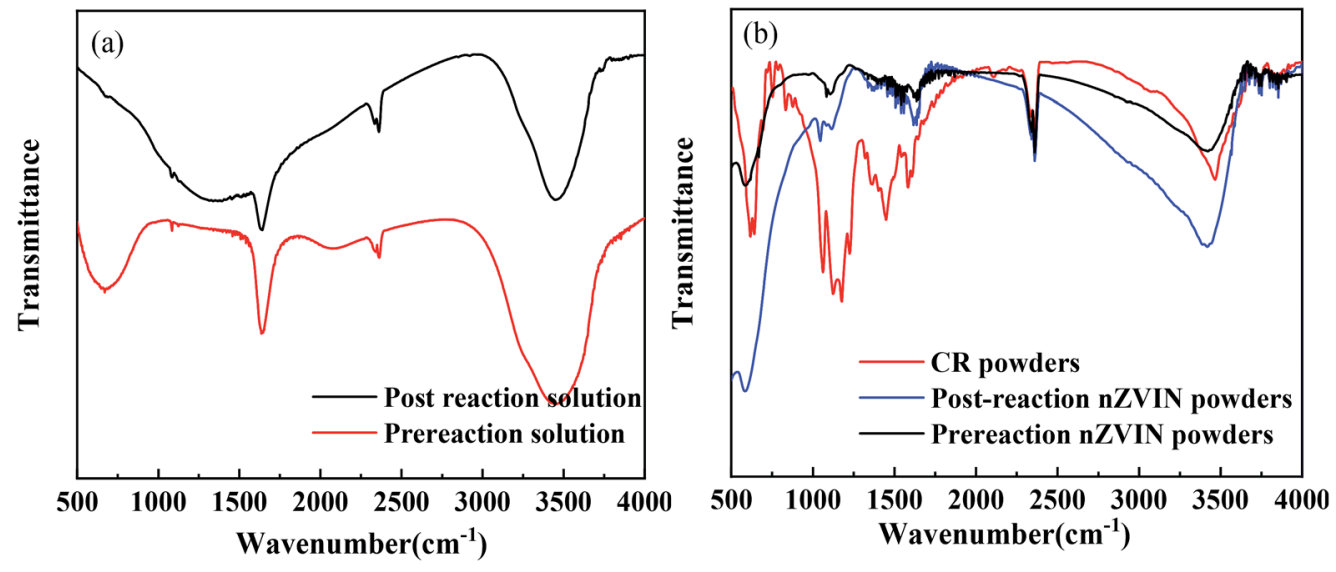

Fig. 8 Concentration-absorbance curve of CR solution. 
solution became colorless after powders were separated by magnetic field (Fig. 6).

The UV spectrum of the solution reaction process is shown in Fig. 7(a). With the increase of nZVIN powders addition time, the absorbance of CR gradually decreased. At $18 \mathrm{~min}$, the CR absorbance curve became smoother, and the characteristic absorption peak $(\lambda=497 \mathrm{~nm})$ disappeared. Therefore, it can be considered that the CR in the solution has been removed after adding the nZVIN powders for $18 \mathrm{~min}$, which indicates that nZVIN powders have a good removal effect on CR.

In view of the great advantages of nZVIN powders in the application of azo dyes, such as excellent magnetic properties and obvious decolorization effects, we have carried out a series of studies on the decolorization effect under different conditions, and conducted a preliminary exploration on the decolorization mechanism.

Firstly, we performed the removal test at different initial concentrations of CR. As shown in Fig. 7(b), the unit removal of CR by nZVIN powders increased with the initial concentration of CR, and increased with the increase of the processing time of nZVIN powders, but grew slowly after $15 \mathrm{~min}$. And the maximum removal of CR by nZVIN powders can reach $140 \mathrm{mg}$ $\mathrm{g}^{-1}$. This exceeds the amount of degradation of common nZVI composites $\left(126 \mathrm{mg} \mathrm{g}^{-1}\right) \cdot{ }^{15}$

The $\mathrm{pH}$ value of the solution is one of the important factors affecting on the treatment effect of sewage. We studied the removal rate of CR by nZVIN powders under different initial solution $\mathrm{pH}$ values (7.0-11.5) of solution. When the initial $\mathrm{pH}$ value was in the range of $\mathrm{pH} 7.0-11.5$, the removal rate of CR by nZVIN powders still maintained above 90\% (Fig. 7(c)). Compared with the current removal rate of nZVI $(86 \%),{ }^{16}$ the common dye wastewater in daily life is generally alkaline. Therefore, a broad ph range is of great significance for the treatment of common dye wastewater in daily life by nZVIN powders.

Fig. 7(d) shows the effect of removing the CR solution by nZVIN powders stored in open air for different times, and the initial concentration of CR is $150 \mathrm{mg} \mathrm{L}^{-1}$. By comparing the CR removal rates, the CR removal ability of nZVIN powders with a storage time of up to one year, has no degradation. Because of the special core-shell structure, the coated ferric oxide can prevent its oxidation and pollution in the air, so that nZVIN powder has a good stability, the use of practical life has a broad prospect.

To further investigate the decolorization mechanism of nZVIN powders, we performed infrared spectroscopy on the sample, as shown in Fig. 8. The absorption peak of the chromogenic group $\mathrm{N}=\mathrm{N}$ bond at $1630 \mathrm{~cm}^{-1}$ and the sulfonic acid group at $620 \mathrm{~cm}^{-1}$, disappeared after nZVIN powders being put in the solution (Fig. 8(a)), which further proves that nZVIN powders have a good treatment effect on azo dye wastewater. The infrared absorption peak of nZVIN powders before and after being put in the CR solution, did not make any differences (Fig. 8(b)), which indicated that nZVIN powders had degraded the CR.

The micro-morphology and structure of nZVIN powders (Fig. 4) and the infrared spectrum of the samples before and after the reaction (Fig. 8), indicates that the decolorization procedures of $\mathrm{CR}$ solution go through two processes of adsorption and degradation. Firstly, because the surface layers of powders have a large number of defects and lattice deformity, nZVIN powders have a strong adsorption effect on $\mathrm{CR}$, and the $\mathrm{CR}$ in the solution are adsorbed on the surface of the powders. Then, the oxidation of zero-valent iron atoms inside nZVIN powders causes atomic hydrogen to be generated in the water, and [Ni] catalyzes the generation of atomic hydrogen, and finally the azo bond of the CR molecule adsorbed on the surfaces of the powders are reductively cracked by the generated atomic hydrogen, and the CR solution is bleached. ${ }^{17}$

\section{Conclusions}

(1) The nZVIN powders were prepared by liquid phase reduction method, which solved the problem that it is difficult to prepare nZVI powders under normal temperature and pressure. The method is simple, efficient and pollution-free. Those nZVIN powders have a unique core-shell structure with a particle size of about $60 \mathrm{~nm}$ and excellent oxidation resistance, which solve the problem that the nZVI powders are easily oxidized and difficult to store.

(2) The nZVIN powders have good magnetic properties and can be separated and recovered by magnetic field treatment, which solve the problem of recycling powders for sewage treatment.

(3) In the decolorization treatment of dye wastewater simulated by Congo red (CR) dye, nZVIN powders can maintain a removal rate of more than $90 \%$ for CR solutions with different $\mathrm{pH}$ values (7.0-11.5) and initial dye concentration (50$200 \mathrm{mg} \mathrm{L}^{-1}$ ). The research results show that nZVIN powders have broad application prospects in the treatment of azo dye wastewater.

\section{Conflicts of interest}

There are no conflicts to declare.

\section{Acknowledgements}

This study was supported by National Natural Science Foundation of China (grant no. 61361008).

\section{Notes and references}

1 P. T. Almazán-Sánchez, I. Linares-Hernández, M. J. SolacheRíos and V. Martínez-Miranda, Water, Air, Soil Pollut., 2016, 227, 100.

2 R. Brindha, P. Muthuselvam, S. Senthilkumar and P. Rajaguru, Chemosphere, 2018, 201, 77-95.

3 S. Dutta, R. Saha, H. Kalita and A. N. Bezbaruah, Environmental Technology \& Innovation, 2016, 5, 176-187.

4 J. Fan, Y. Guo, J. Wang and M. Fan, J. Hazard. Mater., 2009, 166, 904-910.

5 D. V. Kerkez, D. D. Tomašević, G. Kozma, M. R. BečelićTomin, M. D. Prica, S. D. Rončević, Á. Kukovecz, 
B. D. Dalmacija and Z. Kónya, J. Taiwan Inst. Chem. Eng., 2014, 45, 2451-2461.

6 S. Ye, M. Yan, X. Tan, et al., Facile assembled biochar-based nanocomposite with improved graphitization for efficient photocatalytic activity driven by visible light, Appl. Catal., $B, 2019,250,78-88$.

7 S. Ye, G. Zeng, H. Wu, et al., Biological technologies for the remediation of co-contaminated soil, Crit. Rev. Biotechnol, 2017, 37(8), 1062-1076.

8 Q. Huang, M. Liu, L. Mao, D. Xu, G. Zeng, H. Huang, R. Jiang, F. Deng, X. Zhang and Y. Wei, J. Colloid Interface Sci., 2017, 499, 170-179.

9 V. Kecić, Đ. Kerkez, M. Prica, O. Lužanin, M. Bečelić-Tomin, D. T. Pilipović and B. Dalmacija, J. Cleaner Prod., 2018, 202, 65-80.

10 K. V. G. Ravikumar, S. Santhosh, S. V. Sudakaran, Y. V. Nancharaiah, P. Mrudula, N. Chandrasekaran and A. Mukherjee, J. Environ. Chem. Eng., 2018, 6, 1683-1689.
11 A. A. Oladipo, A. O. Ifebajo and M. Gazi, Appl. Catal., B, 2019, 243, 243-252.

12 L. Zhang, Q. Shao and C. Xu, J. Cleaner Prod., 2019, 213, 753761.

13 H. Dong, Z. Jiang, C. Zhang, J. Deng, K. Hou, Y. Cheng, L. Zhang and G. Zeng, J. Colloid Interface Sci., 2018, 513, 117-125.

14 H. Zhao, Z. H. Zhu, X. L. Zheng, C. Xiong and Q. Y. Lin, RSC Adv., 2016, 20, 16413-16418.

15 R. Kvg, S. Das, J. W. Osborne, et al., Novel nano-bio (nano zerovalent iron and Klebsiella sp.) composite beads for congo red removal using response surface methodology, $J$. Environ. Chem. Eng., 2019, 7(5), 103413.

16 G. B. Jegadeesan, S. Amirthavarshini, J. Divya, et al., Catalytic peroxygen activation by biosynthesized iron nanoparticles for enhanced degradation of Congo red dye, Adv. Powder Technol., 2019, 30, 2890-2899.

17 Y. Lin, Z. Chen, M. Megharaj and R. Naidu, J. Colloid Interface Sci., 2012, 381, 30-37. 